\title{
PHARAONISCHE BESCHNEIDUNG
}

\author{
Erika Feucht
}

"Beschneidungen in Mauretanien nehmen zu". So lautet die Überschrift eines Artikels in der Süddeutschen Zeitung vom 11.03.02. Eine Befragung der Welthungerorganisation hat ergeben, dass Beschneidung von Mädchen besonders bei Analphabeten und Menschen, die lediglich die Koranschule besucht haben, dramatisch zugenommen hat. Die Mauretanierinnen werden bereits im Säuglingsalter beschnitten, da ihre Eltern der Meinung sind, durch den Eingriff werde die sexuelle Begierde der Mädchen verringert. Diese Sitte, die vor allem in Afrika ausgeübt wird, führt in den südlichen Ländern zu grausigen Verstümmelungen mit wiederholten Todesfolgen. Da ich als Frau immer wieder, überwiegend von Frauen, nach der Herkunft der Beschneidung, die in ihrer drastischen Form als "pharaonische Beschneidung" bezeichnet wird, gefragt werde, möchte ich dieses Thema noch einmal aufgreifen und aus historischer Sicht beleuchten. Wenn ich auch zu keinen neuen Erkenntnissen gelangen kann, sei mir dennoch erlaubt, die verstreuten Aussagen dazu und Versuche ihrer Interpretation hier vorzulegen und kritisch zu betrachten. Der Vater von drei Töchtern, dessen Interessen zwar im Religiösen zentrieren, doch weit darüber hinausgehen, möge mir erlauben, ihm zu Ehren ein religiös verbrämtes Brauchtum, dessen Ursprung den Pharaonen zugeschrieben wird, hier noch einmal zusammenfassend aufzurollen.

Als Ursprung der weit verbreiteten Sitte der männlichen Beschneidung werden Reinheitsgründe oder kultische Gründe angenommen. Die weibliche Beschneidung basiert auf der Furcht, Frauen könnten Gefallen am Beischlaf finden und voreheliche bzw. außereheliche Beziehungen eingehen.

Seit vorgeschichtlicher Zeit (ca. 3000 v. Chr.) ist in Ägypten männliche Beschneidung sowohl auf Darstellungen sowie bei Mumien nachweisbar und wird in Texten erwähnt. ${ }^{1}$ In allen gesellschaftli-

${ }^{1}$ Es wurde unterschieden zwischen einem Schnitt in der Vorhaut, der ein V entstehen lässt, und der Entfernung der Vorhaut. 
chen Schichten praktiziert, scheint für die Beschneidung kein festes Alter vorgesehen gewesen zu sein, noch wurden alle Männer beschnitten. ${ }^{2}$

Otto Meinardus hat versucht, der Beschneidung eine, auf altägyptischen Vorstellungen beruhende, religiöse Erklärung zu geben. Er ist der Ansicht, dass, wie einige afrikanische Stämme in ihren Mythen, die Ägypter die Vorstellung von einer bisexuellen Seele hatten. Analog zu den ägyptischen Urgöttern, die als Schöpfer allein die Welt und mit ihr die Menschen erschaffen haben, hätte jeder Mensch eine männliche und eine weibliche Seele. Diese beiden Seelen manifestierten sich in den Sexualorganen, wobei die weibliche Seele bei einem Mann in der Vorhaut, die männliche Seele bei einer Frau in der Klitoris verkörpert sei. Daher müssten die das entgegengesetzte Geschlecht enthaltenen Teile vor der Reife entfernt werden. ${ }^{3}$ Allerdings bleibt er den Beweis für seine Behauptungen schuldig und, so faszinierend diese Interpretation auch sein mag, fehlt ihr jede Grundlage in den altägyptischen Quellen. Zwar verkörpern die Urgötter "Vater und Mutter" zugleich, doch ist in den altägyptischen Vorstellungen eine Bisexualität der menschlichen Seele nicht nachzuweisen. Die drei ägyptischen Seelenbegriffe, der den Menschen zu Lebzeiten begleitende Ka, der dem Leichnam in Gestalt eines Vogels mit Kopf des Verstorbenen entfliegende Ba und der im Tod Verklärte (Ach), sind männlichen Geschlechts. Auf Kees ${ }^{4}$ fußend, sieht er in den Lippen (fem.) die weibliche Komponente des Ptah, in den Zähnen (mask.) die männliche, mit der der Gott durch die Zunge den Schöpfungsakt vollbracht habe. Setzt er dies voraus, so ist es jedoch unlogisch, die männliche Seele der Frau nicht allein in der Klitoris sondern auch in den mit ihr zu entfernenden Schamlippen, die, nach dem eben gesagten, die weibliche Komponente beinhal-

${ }^{2}$ Zusammenfassend bereits Alfred Wiedemann, Herodots zweites Buch (Leipzig 1890), 410 ff. Auf neuerem Stand Erika Feucht, Das Kind im Alten Ägypten (Frankfurt-New York 1995), 245ff. mit älterer Literatur.

${ }^{3}$ Otto F.A. Meinardus, "Mythological Historical and Sociological Aspects of Practice of Female Circumcision among the Egyptians", Acta Ethnolol. (Ac. Sci. Hung. 16 (3-6), 387ff.; ders., Christian Egypt. Faith and Life, (Kairo 1970), S. $318 f f$. Paul Ghalioungui, The House of Life, (Amsterdam 1973), 93ff. übernimmt diese Vorstellung, verwechselt aber Ptah mit Chnum und sieht in den Lippen (fem.) des Chnum die Mutter, in seiner Zunge (mask.) den Vater.

${ }^{4}$ Kees, Götterglaube, 291 basierend auf Kurth Sethe, "Das Denkmal Memphitischer Theologie, der Schabakostein des Britischen Museums", UGÄ̈ 10, ${ }^{2}$ Hildesheim 1964), 57 Z. 55. Zähne und Lippen seien die Neunheit, die allerdings mit Same und Hand des Atum zusammen gebracht werden. 
ten, zu sehen. Fehl geht er auch in der, wenn auch vorsichtigen, Andeutung, dass bei einer Beschneidungsszene am Tempel des Chons-das-Kind im Mutbezirk von Karnak aus dem Ende des 2. Jt. v. Chr. möglicherweise die Beschneidung an einem Knaben und einem Mädchen praktiziert werde. Wie wir aus der Parallele, der Beschneidungsszene im Geburtszyklus Amenophis' III., ersehen, handelt es sich um einen Knaben und seine Ka-Seele, die dem gleichen Geschlecht angehört. ${ }^{5}$ Bei den heutigen Fellachen sieht Meinardus eine Erinnerung an die Bisexualität in der Praxis, den Jungen zur Beschneidungszeremonie weibliche Kleider anzuziehen, die sie vor der Beschneidung ablegen. Auch in der Beschimpfung von Jungen als weibisch und von unbeschnittenen Frauen als männlich möchte er einen Nachhall dieser Vorstellung sehen. Doch ist es auch in Kulturen, die keine Beschneidung kennen, weit verbreitet, jemanden durch Zuordnung zum anderen Geschlecht abzuwerten. Zuletzt sei noch darauf hingewiesen, dass jede Erklärung durch die frühen Religionen eine Rechtfertigung des vom Menschen Ersonnenen ist.

Im 5. Jh. v.Chr. berichtet Herodot, dass die Ägypter und die, die es von ihnen angenommen haben, Beschneidung ausübten und zwar aus Gründen der Reinheit. ${ }^{6}$ An anderer Stelle hebt er hervor, dass die schwarzhäutigen Kolcher mit wolligem Haar, die Ägypter und die Äthioper die einzigen Völker seien, die ursprünglich Beschneidung praktizierten. Andere Völker hätten es von den Ägyptern übernommen. Weiter unten fährt er fort: "Doch, was die Ägypter und die Äthioper selbst betrifft, so kann ich nicht sagen, welches Volk es von welchem gelernt hat, denn es beruht auf einem uralten Brauch". ${ }^{7}$ Diese Aussagen werden immer wieder als Beleg für weibliche Beschneidung zitiert. Allerdings ist nur von Beschneidung, einmal von Kindern der Phönizier die Rede, und es bleibt offen, ob Herodot sich auf die Beschneidung beider Geschlechter oder nur auf die der Männer bezieht.

${ }^{5}$ Nagel, in: AnOr 20, 1952, 94ff; de Jong, "De tempel van Chonsoe-het-kind", in: Ibis 8, 1983, $106 \mathrm{f}$ mit Abb. 33 u. 34. Zu Amenophis III. vgl. Hellmut Brunner, "Die Geburt des Gottkönigs", $\ddot{A A} 10,1964,157 f f .162$ u. 193, Tf. 15, Sz. XV L.

${ }^{6}$ Herodot II, 36 f.: "Die Geschlechtsteile lassen die anderen Völker wie sie sind; nur die Ägypter beschneiden sie."

${ }^{7}$ II, 104. 
In arabischen Ländern wurde die Beschneidung beider Geschlechter bereits in vorislamischer Zeit praktiziert, wird aber nur teilweise von den Muslimen übernommen. Wird die männliche Beschneidung durch die Sunna gefordert, so ist die weibliche Beschneidung fakultativ und daher nicht Brauch in allen islamischen Ländern. Nach der Überlieferung habe Mohamed einer bekannten Beschneiderin von Frauen, Umm Attia, gesagt, sie solle nur die Spitze beschneiden, da das besser sei für die Frauen. ${ }^{8}$ Einer Konvertierten, die ihn fragte, ob sie sich beschneiden lassen solle, rät er davon ab, da es schmerzhaft für die Frau, angenehm für den Mann sei. ${ }^{9}$ In Arabien und den östlichen islamischen Ländern ist Frauenbeschneidung unüblich. Sie ist hauptsächlich auf Ägypten, den Sudan und die zentralafrikanischen Länder beschränkt.

Im Niltal hat Rudolf Herzog verschiedene Praktiken der weiblichen Beschneidung beobachtet. ${ }^{10}$ Im Delta bis herunter nach Edfu ist es Brauch, die Klitoris oder nur einen Teil von ihr herauszuschneiden. Südlich von Edfu bis in die Gegend von Wadi Halfa werden neben der Klitoris auch die inneren Schamlippen (labia minora) entfernt, während südlich von Wadi Halfa, in der sogenannten sudanesischen Beschneidung, die äußeren Schamlippen (labia majora) mitbeseitigt werden. Anschließend wird die Wunde zusammengenäht und nur eine kleine Öffnung, manchmal eine zu kleine Öffnung, zum Urinieren und Ausbluten während der Menstruation

8 Mahmoud Karim Roshdi - Ammar, Female Circumcision and Sexual Desire, (Kairo 1965), 4.

${ }^{9}$ Op. cit., 120.

${ }^{10}$ Rudolf Herzog, Die Nubier, (Berlin 1957), 100. Ingrid Bierer-Lieselotte Walther, "Ritualbeschneidung an Mädchen", Nürnberger Blätter zur Archäologie, (Sudan, Sonderheft 1999), Fs. Wenig, 139 unterscheiden zwischen 1. Rituellem Einritzen, Einstechen oder Entfernen der Vorhaut der Klitoris und teilweise oder komplette Entfernung der Klitoris, 2. Entfernen der gesamten Klitoris sowie der kleinen Schamlippen, 3. Entfernen der Klitoris und inneren Schamlippen sowie Ausschälen der Innenschichten der äußeren Schamlippen und Zusammennähen ( sog. "pharaonische Beschneidung", in Nubien fast ausschließlich praktiziert). Karim-Ammar, op.cit., 6 unterscheidet zwischen entfernen der 1. Spitze der Klitoris und der inneren Schamlippen, 2. Teil der Klitoris und inneren Schamlippen, 3. Gesamte Klitoris und inneren Schamlippen, 4. Ganze Klitoris, inneren Schamlippen (Sudan). Mit Darstellungen letztlich Mary Knight, "Curing Cult or Ritual Mutilation", Isis 92, 2001, 317ff., bes. 320ff. mit weiterer Literatur in Anm. 12. Auf S. 329 mit Anm. 26 weist sie auf die Untersuchung von Muhammad Fayyad, Al-batr al tanasuli li-l-inath, (Kairo 1998), 140f. hin, dass Mitte der 90iger Jahre des 20. Jh. 64 Prozent der Beschneidungen von Männern (Ärzten, Barbieren und Schlächtern), 38 Prozent von Hebammen vorgenommen wurden. 
belassen, was häufig zu Entzündungen führt. Vor der Hochzeit muss eine zu klein geratene Öffnung wieder aufgeschnitten werden. Nach der Geburt eines Kindes, bei längerer Abwesenheit des Mannes oder bei Witwen kann sie wieder geschlossen werden. Diese Art der Beschneidung wird im Sudan "Pharaonische Beschneidung" genannt. Wie es zu dieser Bezeichnung gekommen ist, liegt im Dunkeln. Auch ist nicht klar, woher diese Sitte kommt.

Beschneidung von Mädchen scheint in altägyptischer Zeit entweder nicht erwähnenswert oder nicht üblich gewesen zu sein. Sie ist anthropologisch nicht nachgewiesen und auch an den ausgetrockneten Mumien schwer festzustellen. G. Maspero hat bei der Untersuchung. der Mumie der in der 17. Dynastie, d.h. der zweiten Hälfte des 16. Jh. v. Chr., gestorben Gemahlin des Königs Ahmose I., Inhapi, eindeutig feststellen können, daß sie keine Beschneidung aufweist. ${ }^{11}$

Ein Sargtext aus dem frühen 2. Jahrtausend v. Chr., der auf Särgen des mittelägyptischen el-Berscheh aufgezeichnet ist, wird immer wieder als erster Hinweis dafür aufgeführt, daß bereits zu dieser Zeit weibliche Beschneidung in Ägypten Brauch war. Hier heißt es, dass man sich zur Zauberpraxis des Belebens mit dem Ausfluss (? bid $)^{12}$ eines Mädchens ${ }^{13}$, das ' $m$ ' $t$ ist, und der Flüssigkeit (?) eines Kahlköpfigen, der ' $m$ ' ist, einreiben solle. ${ }^{14}$ ' $m$ ' wird sowohl in der männlichen wie weiblichen Form mit dem Phallus mit Ausfluss determiniert und daher in der ägyptologischen Literatur mit "unbeschnitten" übersetzt. In medizinischen Texten des späteren Mittleren Reichs und des Neuen Reichs begegnet uns die Verwendung eines solchen Ausflusses (?) von Männern und Frauen, die ' $m$ ' $(t)$ sind, ${ }^{15}$ als Salbmittel wieder. ${ }^{16}$

${ }^{11}$ Gaston Maspero, Les Momies royales de Deir el-Bahari, Mémoires de la Mission Archéologique Française au Caire I, (Paris 1889), S. 533.

${ }^{12}$ Wort unbekannter Bedeutung, s. Dimitri Meeks, Année Lexicographique II (1978), (Paris 1981), 121.

${ }^{13}$ Von mir fälschlicherweise hrdt gelesen (Feucht, op. cit., 249). Deutlich geht jedoch aus den Parallelen B9C und B4C die Lesung jdt bzw. $<j d>j j t$ hervor. Sowohl hrdt wie auch $j d t$ kann ein bereits reifes, junges Mädchen bezeichnen ( $\mathrm{Wb}$ I, 151 u. Feucht, op. cit., 515 u. 539).

${ }^{14}$ CT VII, 450 d. Herman Kees, Totenglaube und Jenseitsvorstellungen des Alten Ägypter, (Leipzig 1926), 447. Feucht, Kind, 249 mit älterer Literatur. Saphinaz-Amal Naguib, in: BSEG 7, 1983, 79ff. kommt auch zu keinem neuen Ergebniss.

${ }^{15}$ Erkrankung eines inneren Organs wird behandelt mit Urin einer ' $m$ ' $t$ (det. mit Phallus mit Ausfluss und der sitzenden Frau): pmed.Berlin 3038, 6, 1. 6, 4. 2, 8, eine Verletzung mit dem Ausfluß ( $m t w t=$ Same) eines ${ }^{\prime} m$ ' $m$ und einer ' $m{ }^{\prime} t$ : pEbers 88, 7. S. Hildegard v. Deines und Hermann Grapow, Grundriß der Medizin der Alten Ägyter IV, 1, 152. 155. 213. 
Noch zweimal ist dieser Ausdruck belegt. Um 730 v. Chr. betreten die Fürsten und Könige des Nordens, die in einer Allianz gegen Pianchi, Herrscher über die Thebais nubischer Abstammung, nach Oberägypten vorgedrungen waren und, von diesem nach Memphis zurückgedrängt, sich ihm unterworfenen haben, nicht das Haus des Königs, da sie ' $m$ ' seien und Fisch äßen. ${ }^{17}$ "Nemaret hingegen trat ein, da er rein sei und keinen Fisch äße." Breasted war bei der Übersetzung von ' $m$ ' sehr vorsichtig. Er übersetzt "unclean". ${ }^{18}$ Während Budge in der männlichen Form "man suffering from some defect of the sexual organ" sieht, ${ }^{19}$ was alles beinhalten kann, gibt Ebers es mit "unbeschnitten" wieder ${ }^{20}$ eine Übersetzung, die das Wörterbuch beim Substantiv "Unbeschnittener" mit Fragezeichen übernimmt. Grimal entscheidet sich für "unbeschnitten". ${ }^{21}$ Auch Clère kommt, nach langer Diskussion, zu diesem Ergebnis. ${ }^{22}$ Durch den Phallus hat das Wort ' $m$ ' eindeutig eine sexuelle Konnotation. ${ }^{23}$ Gehen wir von der Übersetzung "unbeschnitten" aus und sehen in den Fürsten Unbeschnittene, dann müssen wir annehmen, dass es sich bei der männlichen Beschneidung um eine in Ägypten nicht allgemein übliche Sitte handelte, während sie bei den in kultischen Vorschriften besonders strengen Nubiern zum Reinheitsgebot gehörte, wes-

Mit dem Urin oder Ausfluß einer Frau, die ' $m$ ' $t$ ist, wird die Entzündung bzw. das Feuer gelöscht: pmedBerlin $60(5,12-6,2$, vgl. auch 9, 5-7). $64(6,4)$ und pBM 10188, 29,15 = Apophisbuch III. Zusatz. S. v. Deines-Grapow, Grundriß $\mathrm{V}, 265 f$.

${ }^{16}$ WB. I, 185, 11 pKahun 7, (1, 24-25):Es werden die Füße einer Frau mit dem ' $m$ ' $t$ (det. mit dem nw-Topf und Pluralstrichen) eingerieben $\left({ }^{\prime} m\right.$ ' , det. mit schlagendem Mann, vgl. auch ' $m$ ' $m$ det. mit schlagendem Arm: einreiben: eingerieben werden die Füße des ermüdeten Dedi im p.Westcar 7, $\left.17 \mathrm{c}^{\mathrm{\prime}} \mathrm{m} \mathrm{m}\right)$. Vgl. auch ' $m$ ' $t$ als Einreibemittel im pEbers 67, 17, das pEbers 482a schwarz ist (s. v.DeinesGrapow, Grundriß VI, 90.91. 94 und 292; VII, 1, S. 140.

17 Nicolas C. Grimal, La stèle triomphale de Pi('nkh)y au Musée du Caire," Memoires de l'Institut Francais d'Archéologie Orientale du Caire 105, 1981, 176 mit Anm. 529.

${ }^{18}$ BAR IV, $443 \S 882$ und Anm. d.

${ }^{19}$ Sir E.A. Wallis Budge, Egyptian Hieroglyphic Dictionary I, (London 1920), 122a führt auch die fem. und die mask. Pl.-form auf.

${ }^{20}$ Georg Ebers, Ägypten und die Bücher Mose's, (1868), 233 u. 284.

${ }^{21}$ Jacques J. Clère, Les Chauves d'Hathor, OLA 63, (Leuven 1995), 17 ff.loc. cit.

22 Loc. cit.

${ }^{23}$ Wb I, 185, 13 mit der Pianchistele als Belegstelle. Raymond O. Faulkner, Concise Dictionary of Middle Egyptian, (Oxford 1964), 42 läßt den Sargtext außer Betracht und erwähnt nur ' $m$ ', det. mit schlagendem Mann und ' $m$ ', det. mit schlagendem Arm, als "einreiben". 
wegen von dem nubischen Pianchi Unbeschnittene als unrein betrachtet wurden.

In einem Text aus der Mitte des 1. Jahrtausends v. Chr. kommt das Wort noch einmal im Gegensatz zu "rein sein" vor. In den Reinheitsvorschriften des an der Grenze zu Nubien auf der Insel Philae liegenden Isistempels ist denen, die ' $m$ ' sind, das Betreten des Tempels verboten. Es werden bestimmte Pflanzen, Esel, Hund, ' $m$ ' und Ziegen aufgezählt, die den Tempel nicht betreten dürfen. ${ }^{24}$ Auch hier wird Bezug auf eine Unreinheit genommen, die mit Hinweis auf das Determinativ und die Parallele auf der Pianchistele ebenfalls mit "unbeschnitten sein" übersetzt wird. Ob zu dieser Zeit alle Priester beschnitten sein mussten, ist nicht belegt. Erst aus den Beschneidungsurkunden aus der zweiten Hälfte des 2. Jahrhunderts n. Chr. geht hervor, dass Priesteranwärter sich beschneiden ließen. Um die Erlaubnis zur Beschneidung zu bekommen, mussten sie den Nachweis ihrer Abstammung aus priesterlichem Geschlecht beibringen und eine Prüfung der Makellosigkeit bestehen. ${ }^{25}$ Laien war es bis zu dem Edikt Hadrians, der ein Beschneidungsverbot für alle außer Priester erließ, offensichtlich freigestellt, ob sie sich beschneiden ließen oder nicht. ${ }^{26}$ Ist auch männliche Beschneidung seit vorgeschichtlicher Zeit in Ägypten belegt, so weisen doch, soweit untersucht, nur einige Königsmumien Beschneidung auf. ${ }^{27}$ Doch Pharao war Oberpriester aller Gottheiten! War Beschneidung daher fakultativ oder lokal gebunden? Die von Pianchi nicht vorgelassenen Fürsten stammten aus dem Norden Ägyptens, waren vermutlich Nachkommen der libyschen Dynastie. War es bei ihnen nicht Sitte, sich beschneiden zu lassen? Nemaret hingegen war Fürst von Hermopolis, das in Mittelägypten

${ }^{24}$ Das $m$ wird hier mit der $m$ '-hacke geschrieben. Doch bereits Junker hat darauf hingewiesen, das diese in der Ptolemäerzeit für $m$ stehen kann (Herman Junker, Vorschriften für den Tempelkult in Philä Analecta Biblica 12, (Rom 1959), 152f. u. Anm. S. 155).

${ }^{25}$ Walter Otto, Priester und Tempel im Hellenistischen Ägypten I, (Leipzig-Berlin, 1905), 214 und II, 1908.

${ }^{26}$ Antoninus Pius hat es den Juden wieder gestattet ( Schürer, Geschichte des Jüdischen Volkes im Zeitalter Fesu Christi $\mathrm{I}^{3}$, Leipzig 1901, 675 ff.

${ }^{27}$ Die Mumien Amenophis' II. und Thutmoses' IV. sollen Beschneidung aufweisen, möglicherweise auch die Ramses' IV. und V., andere Königsmumien, soweit feststellbar, nicht. S. hierzu de Wit, Z̈̈S 99, 1972, 47, T. Smith, BdE V,1, 1907, 225, ders., ASAE 4, 1904, 112. Der 5-6jährige Prinz Sapai aus der 18. Dyn. scheint beschnittwen gewesen zu sein (G.Elliot Smit - Warren R. Dawson, Egyptian Mummies, (London 1924), 93), der 1 ljährige Sohn Amenophis' II. hingegen nicht (op. cit., 25).S. a. Feucht, Kind, 247f. mit Anm. 1240 ff. 
liegt. Die Särge, auf denen der oben besprochene Sargtext aufgezeichnet ist, stammen alle aus el-Berscheh, d.h. aus Mittelägypten. Möglicherweise lag hier eine Brauchtumsgrenze. Eine Antwort können nur Mumienuntersuchungen geben. Sollte Beschneidung bei Libyern oder in Unterägypten nicht üblich gewesen sein, können wir in den von Pianchi nicht empfangenen Fürsten und Königen Unbeschnittene sehen, die der aus dem Süden stammende Eroberer als unrein ansah.

Allerdings geht die Übersetzung "unbeschnitten" allein auf den Phallus als Determinativ zurück, mit dem sowohl ' $m$ ' wie ' $m$ ' $t$ geschrieben wird, und die Tatsache, das bei Pianchi ' $m$ ' im Gegensatz zu "rein sein" verwendet wird. Bei Pianch ${ }^{28}$ und in der Inschrift auf Philae hat der Phallus keinen Ausfluss. Doch warum wird ein Unbeschnittener bzw. eine Unbeschnittene sowohl im Sargtext als auch in einigen medizinischen Texten ${ }^{29}$ mit dem Phallus, aus dem etwas herausfließt determiniert? Wenn es sich um Unbeschnittene handelt, kann kein Blut wie beim Beschneiden geflossen sein. ${ }^{30}$ Selbst bei diesen heilt die Wunde gewöhnlich ohne Ausfluss als Nebenwirkung. Das kann also nicht gemeint sein. Zu medizinischen Zwecken zum Einreiben verwendet, muß es sich um eine flüssige, wohl schmierige Substanz aus den Genitalien handeln. Die Fürsten, die von Pianchi nicht empfangen wurden, da sie ' $m$ ' waren, galten als unrein. In den medizinischen Texten wird der als Salbmittel verwendete Ausfluss der ' $m$ ' $(t)$ als Urin, als Samen und einmal als schwarz bezeichnet. Bei Harnröhrenentzündungen können alle drei Formen vorkommen. ${ }^{31}$ Der Ausfluss kann hell (wie der "Samen"), wässrig (wie "Urin") oder eitrig-blutig ("schwarz") sein. Doch können wir nicht davon ausgehen, dass alle Fürsten des Nordens, die Pianchi ihre Ehrerbietung

${ }^{28}$ Deutlich Grimal, op. cit., Z. 150 auf Tf. IVB und XII.

29 pBerlin P 3038, 6, 2,7-9 gefolgt von einer Buchrolle und Pluralstrichen. In anderen Texten kann als Determinativ der einfache Phallus (pBerlin P 3038, 6,35. 5, 12-6, 2) oder ein Topf mit Pluralstrichen (pKahun, Medic. 1, 24-26. pEbers

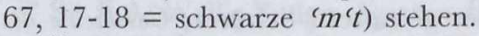

${ }^{30}$ Vgl. Naville, Tb. 17, 29: "das Blut, welches floß vom Phallus des Re, nachdem er sich verwundet $\left(\check{s}^{\prime} t\right)$ hatte." Ob hier jedoch Beschneidung gemeint ist, ist unklar.

${ }^{31}$ z.B. die durch Geschlechtsverkehr übertragene Harnröhrenentzündung (Urethritis). Bei der unspezifischen Form (Erreger Chlamydien oder Mykoplasmen) ist der Ausfluß meist hell (wie Same ?) oder klar (wie Urin ?). Bei der durch Gonokokken verursachten Form der Gonorrhoe (Tripper) ist er eitrig oder eitrigblutig (schwarz ?). Für diese Auskunft danke ich Jürgen Putz. 
erweisen wollten, an einer Geschlechtskrankheit litten. Deswegen sollten wir nach einer anderen Erklärung suchen.

Möglicherweise hat das Wort eine ganz andere Bedeutung als in der ägyptologischen Literatur angenommen. Durch den Phallus als Determinativ wissen wir, dass es eine sexuelle Bedeutung haben muss, die für Männer wie Frauen gilt.

Da wir nicht mit Sicherheit sagen können, ob Priester bereits in pharaonischer Zeit beschnitten sein mussten, kann in dem Sargtext von einem beliebigen Mädchen und einem beliebigen Kahlköpfigen die Rede sein. Voraussetzung war, daß beide ' $m$ ' waren. Doch "Kahlköpfiger" ist seit dem Neuen Reich auch eine Bezeichnung für einen Priester der Hathor oder einer mit ihr verschmolzenen Göttin. ${ }^{32}$ Hathor war die Göttin der Liebe, die sich um die Belange der Frauen kümmerte. Auffallend ist, daß einige ihrer Priester sich als kahlköpfige Hockerstatuen verewigen ließen. In den auf diesen Hockerstatuen verzeichneten Texten wenden sich die Priester vor allem an Frauen. Sie bitten sie um eine Spende und beteuern, dass sie sich für sie einsetzen werden. ${ }^{33}$ Einer beteuert, er werde sich um alle Frauen kümmern, der Witwe einen Gatten geben und eine Aussteuer dem jungen Mädchen. ${ }^{34}$ Ein anderer verspricht dem jungen Mädchen einen Gatten. ${ }^{35}$ Ein dritter droht Frauen, die ihm nichts geben, dass sie allein schlafen und keinen Gatten bekommen werden. ${ }^{36}$ Wieder einer hilft der Gebärenden, gibt einen Mann der Witwe, dem jungen Mädchen einen Geliebten und gibt derjenigen einen Sohn, die darum bittet. ${ }^{37}$ Der nächste hört die Bitte jeder Tochter, die weint und die Hathor versorgt, ${ }^{38}$ und der letzte gibt dem jungen Mädchen einen Gatten und der Witwe Nahrung. ${ }^{39}$ Sollten wir in diesen Versprechen vielleicht mehr als nur eine Fürsprache bei der Göttin der Liebe sehen? Es gibt verschiedene Lexeme für begatten im Altägyptischen. ${ }^{40}$ Die

${ }^{32}$ Jacques J. Clère, Les Chauves d'Hathor, OLA 63, (Leuven 1995). Als Name bereits seit dem Alten Reich üblich, tritt uns der "Kahlköpfige" als Bezeichnung jemandes im Gefolge der Hathor erstmals auf dem ersten illustrierten Papyrus aus der ersten Hälfte der 18. Dynastie, dem Papyrus des Nu, im Tb. 103 entgegen (op. cit., 68, d. Die Varianten sind dort zusammengestellt).

${ }^{33}$ Op. cit., 74 ff.: Doc. A, Z. 12 f.

${ }^{34}$ Op. cit, 110 f., Doc. F, Z. $6 f$

35 Op. cit., 126 f., Doc. I, Text B, Z. 5. 142 ff., Doc. K, Z. 6

${ }^{36}$ Op. cit., $154 \mathrm{f}$ f., Text C.

37 Op. cit., 165 f., Doc. O, Text A, Z. 5 ff.

38 Op. cit., 202 ff, Text A, Z. 3.

39 Op. cit., 209, Doc. GG Z. 4 f.

${ }^{40} \mathrm{~S}$. Wb VI s.v. begatten, Beischlaf, Beischlaf vollziehen. 
meisten von ihnen haben als Determinativ den Phallus mit Ausfluss, d.h. sie zeigen den Samenerguss. Betrachtet man sie jedoch genauer, so stellt man fest, dass sie sich in ihrer Bedeutung unterscheiden. Sollten uns in ' $m$ ' eine weitere Variante vorliegen, die sich auf einen außerehelichen Beischlaf bezieht?

Im Text von Philae wird denen, die ' $m$ ' sind, das Betreten des Tempels verboten. Sie werden mitten unter den Tieren aufgezählt, die den Tempel nicht betreten dürften. Warum werden die ' $m$ ' hier unter den unreinen Tieren aufgeführt? Sollten hier unbeschnittene Tiere gemeint sein? Das hieße, dass nur kastrierte ${ }^{41}$ Tiere zugelassen waren. Doch sollten wir eher davon ausgehen, dass gerade die kastrierten Tiere wie in Judäa ${ }^{42}$ als unrein galten und nicht als Opfergaben zugelassen waren. Es muß sich folglich um als unrein angesehene Menschen handeln. Da zwar Priester in griechisch-römischer Zeit der Beschneidung unterworfen waren, jedoch, wie oben ausgeführt, die Mumien von Pharaonen nur in einigen Fällen Beschneidung aufweisen und nichts darauf deutet, dass die ptolemäischen Könige im 1.Jh. v. Chr. beschnitten waren, Könige als Oberpriester jedoch jederzeit Zugang zu den Tempeln hatten, können wir nicht davon ausgehen, daß unbeschnittene Menschen keinen Zugang zum Tempel hatten. Wir müssen die Bedeutung also woanders suchen.

Herodot berichtet beispielsweise, dass sogar Laien zu Waschungen verpflichtet waren, ehe sie nach einem Beischlaf den Tempel betraten, ${ }^{43}$ ein auch heute noch üblicher Brauch bei den Muslimen. In 5. Mose, 23, 9 ff. lesen wir: "Wenn du in Kriegslager wider deine Feinde ausziehst, so hüte dich vor allem Ungehörigen. Ist jemand in deiner Mitte, der infolge eines nächtlichen Begegnisses nicht rein ist, so soll er vor das Lager hinausgehen [und] nicht [wieder] ins Lager hineinkommen. Gegen Abend sodann wasche er sich mit Wasser, und wenn die Sonne untergeht, darf er [wieder] ins Lager hineinkommen." Deutlich wird hier derjenige, der den Beischlaf vollzogen hat, als unrein und damit als Unglücksbringender angesehen. Erst eine Waschung konnte die Gefahren, die von ihm ausgingen, bannen. War dies ein über Israel hinaus gehender Glaube, und kannte Pianchi, der sich auf einem Feldzug befand, einen ähnlichen Glauben? Hat er deswegen die unterägyptischen Fürsten nicht

\footnotetext{
${ }^{41} s$ c $b$ Wb. IV,43,11 seit MR für Kastrieren von Stieren. S.a. IV, 81,16: $s b$.

42 3. Mose 22, 24. Vgl. auch 5. Mose, 23, 1.

${ }^{43}$ II, 64.
} 
empfangen? Handelt es sich daher bei dem Wort ' $m$ ' um die sexuelle Unreinheit nach dem Beischlaf? Das Determinativ könnte in diese Richtung deuten. Doch warum haben sich dann die unterägyptischen Fürsten nicht einer Waschung unterzogen, bevor sie Pianchi ihre Ehrerbietung erbringen wollten? Kannten sie diese Gewohnheit nicht? So unbefriedigend diese Erklärung ist, ist es nicht auszuschließen, dass die Bedeutung in dieser Richtung zu suchen ist. In diesem Fall müssten wir den Sargtext als ersten Beleg für die weibliche Beschneidung ausschließen.

Die erste eindeutige Aussage zur Beschneidung von Mädchen in Ägypten stammt aus dem Jahr 163 v. Chr. Aus einem griechischen, im Serapeum von Memphis nahe dem heutigen Kairo gefundenen Schriftstück erfahren wir, dass sich eine Frau mit griechischem Namen das von ihrer Tochter im Tempel deponierte Geld erschlichen hat mit der Begründung, ihre Tochter habe das Alter erreicht, " in dem es bei den Ägyptern Sitte sei, zu beschneiden". "Sie benötige das Geld, um ihr neue Kleider und eine Mitgift zu besorgen, d.h. das Mädchen hatte die Reife und damit das heiratsfähige Alter von 13 - 14 Jahren erreicht.

Auch St. Ambrosius erwähnt im 4. Jh. n. Chr. das vierzehnte Lebensalter als Beschneidungsalter für beide Geschlechter: “... die Ägypter beschneiden ihre Männer in ihrem 14. Jahr, und die Frauen unter ihnen werden zur Beschneidung im gleichen Jahr gebracht, denn es ist klar, dass von diesem Jahr an die Lust der Männer zu brennen beginnt und die Menstruation der Frauen beginnt." 45

Strabo, der Ägypten 25-24 v. Chr. besucht hat, ${ }^{46}$ unterscheidet zwischen "umschneiden" bei Jungen und "ausschneiden" bei Mädchen, wie es bei den Juden Sitte sei, ${ }^{47}$ die der Abstammung nach Ägypter seien. ${ }^{48}$ Obwohl Hadrian in der 2. Hälfte des 2. Jh. n. Chr. ein allgemeines Beschneidungsverbot erlassen hat, aus dem Priester

44 F.G.Kenyon, Greek Papyri in the British Museum I, London 1893, S. $32 \mathrm{Nr}$. XXIV, Z. 12.

45 de Patre Abrahamo LVII, Cap. 11, 78(K. Schenkl, Corpus Ecclesiasticorum Latinorum, Wien 1897, 32). Vgl. auch Philo Judaeus zu Genesis 3,47 (R. Marcus, Philo, Questions and Answers on Genesis, Translated from the Ancient Armenian Version of the Original Greek, Cambridge. Mass. 1953).

${ }^{46}$ gelebt 63 v. Chr. bis 20 n.Chr.

47 Geographica XVII.2.5. Vgl. auch XVI.4.9.Vgl. auch Leviticus 12.3.

${ }^{48}$ Op. cit., XVI.2.34 f. Die Juden seien eine Mischung aus ägyptischen, arabischen und phönizischen Stämmen. Die Vorfahren der Judaer seien Ägypter, da Moses ein ägyptischer Priester war. 
ausgenommen waren, scheint es später wieder praktiziert worden zu sein. ${ }^{49}$ Soranus, der im 2. Jh. n. Chr. gelebt hat, wird die erste Beschreibung der Entfernung der Spitze der übergroßen Klitoris bei Mädchen zugeschrieben. ${ }^{50}$ Der byzantinische Hofarzt Aetius von Amida, der in Alexandrien Medizin studiert hat, schreibt im 6. Jh. n. Chr.:" ... bei manchen Frauen wird die Klitoris größer und wird unziemlich und schamvoll. Da sie jedoch ständig durch die Kleider gerieben wird, erregt es sie und lässt den Wunsch nach Beischlaf entstehen. Wegen dieser zunehmenden Größe entschlossen sich die Ägypter, sie zu entfernen, besonders in der Zeit, wenn die Mädchen bereit zur Heirat seien. Der Eingriff wird dermaßen vorgenommen. Sie lassen das Mädchen auf einen Hocker Platz nehmen, und ein starker, hinter ihr stehender Mann legt seine Vorderarme unter ihre Schenkel und ihr Hinterteil, damit ihre Beine und ihren ganzen Körper festhaltend. Der vor ihr stehende Operateur ergreift mit einer breiten Pinzette ihre Klitoris und zieht sie mit seiner linken Hand heraus, während die Rechte sie mit den Zähnen der Pinzette abschneidet". 51 Paulus Aeginetes (VI,70) gibt an, man solle die Frau zur Beseitigung der Klitorishypertrophie in die Rückenlage geben. In beiden Fällen ist nur von der Beschneidung der Klitoris die Rede. Ein bestimmtes Alter ist hierfür nicht festgelegt. Wird sie von Ärzten vorgenommen, so beschränken sich diese häufig auf die Entfernung der Klitorisspitze. Auf dem Land werden jedoch meist die ganze Klitoris und gelegentlich auch Teile der inneren Schamlippen entfernt. Die im Inneren Afrika praktizierte Entfernung der inneren und äußeren Schamlippen und das Zunähen ist in Ägypten nicht Brauch.

Im Gegensatz zu Herodot, der behauptet, die anderen Völker hät-

${ }^{49}$ Antininus Pius hat es den Juden wieder gestattet (Walter Otto, Priester und Tempel im Hellenistischen Ägypten, Leipzig-Berlin 1908, 279.

${ }^{50} \mathrm{Zu}$ dieser Überlieferung und den ihr folgenden vgl. ausführlich M. Knight, op. cit., $322 \mathrm{ff}$. Galen, der ebenfalls im 2. Jh. gelebt hat, wird ein Text zugeschrieben, in dem er schreibt, die übergroße Klitoris sei von den Ägyptern als unschicklich angesehen und wurde beschnitten. (Introd. 10, Bd. XIV, S. 706k. S. hierzu K.G. Kühn, Hgg. Medicorusm graecorum opera quae extant, Leipzig 1921 ff.)

${ }^{51}$ Biblia Iatrika XVI, 106. J.V. Ricci, Aetios of Amida The Gynecology and Ostetrics of the VIth Century A.D., Translated from Cornarius' Text of 1542, (Philadelphia 1950), 5ff. S.a. Knight, op.cit. 327. Theodor Hopfner, Das Sexualleben der Griechen und Römer von den Anfängen bis ins 6. Jahrhundert nach Christus, (Prag 1938), 223 verweist auf Stein bei Rohleder III, 279, der die Skozen in Russland erwähnt, die als "erste Reinheit" die kleinen Schamlippen meist mit der Klitoris ausschnitten, worauf als "zweite Reinheit" das Abschneiden und Abbrennen der Brüste folgte. 
ten die Beschneidung von den Ägyptern übernommen, ${ }^{52}$ gab es im Mittelalter das Bestreben, sowohl bei den christlichen Kopten Ägyptens wie bei den Muslimen die weibliche Beschneidung auf das Judentum zurückzuführen. Michael, koptischer Metropolit von Damiette erklärt sie im 12. Jh. n.Chr. folgendermaßen: Da Hagar von Frauen, die ihren Sohn Ismael heiraten wollten, die Beschneidung verlangt habe mit den Worten: "Wir sind ein beschnittenes Volk, beide, unsere Männer und Frauen; und wir heiraten nur die, die uns gleich sind" hätten die Frauen sich beschneiden lassen. Danach sei die Sitte nach Ägypten gelangt, ${ }^{53}$ denn Ismael heiratete eine Ägypterin. ${ }^{54}$ Ibn Abd el-Hakim berichtet von einer muslimischen Überlieferung, in der die weibliche Beschneidung mit Hagar in Verbindung gebracht wird. Hier heißt es, Sarah habe einen Streit mit Hagar gehabt und in ihrer Eifersucht geschworen, dass sie Hagar sexuell entstellen werde. Auf Abrahams Protest hin antwortete sie, sie habe vor Gott geschworen, dass sie es tun werde und lasse sich nicht in Grottes Auge zur Lügnerin abstempeln. Abraham gab nach und deutete Sarah an, sie könne Hagar beschneiden. So wurde die Beschneidung für Frauen bei den Juden üblich. ${ }^{55}$ Interessanterweise wird sowohl bei den Kopten wie bei den Muslimen die weibliche Beschneidung auf Hagar, die Magd Abrahams, zurückgeführt und als jüdische Sitte erklärt. Außer den jüdischen Äthiopiern praktizieren die Juden jedoch die weibliche Beschneidung nicht. Hagar war aber Ägypterin; an ihrem Sohn Ismael hat Abraham erstmals die Beschneidung vollziehen lassen. ${ }^{56}$ Sollte hier von den Kopten ein Brauch, der in ihrem Land bereits vor dem Christentum praktiziert wurde, über das Judentum legalisiert werden, und haben die Muslime diese Tradierung übernommen?

52 II, 104.

53 O.H.E. Burmester, "The Sayings of Michael, Metropolitan of Damietta" Orientalia Periodica II, 1-2, (1936), S. 123; zitiert nach Meinardus, Christian Egypt, 237.

54 1. Mose 21, 21

55 Ibn 'Abd Al-Hakim, The History of the Conquest of Egypt, North Africa and Spain, Known as the Futuh Misr, hgg. von Charles C. Torrey, (New Haven 1922), S. 11: vgl. auch Al -Tabari (Beirut ${ }^{3}$ 1992), 1, S. 130; zitiert von Sami A. Aldeeb AbuSahliehe, "Jehova, his Cousin Allah, and Sexual Mutilations", Sexual Mutilations a Human Tragedy, hgg. Von George C. Denniston und Marilyn Fayre Milos, (New York-London 1996), S. 48.

56 1. Mose 17, 23. 


\section{Fazit}

Mit Sicherheit können wir sagen, dass es in der Mitte des 2. Jahrhundert v. Chr. in Ägypten Sitte war, auch Mädchen zu beschneiden. $\mathrm{Ob}$ dieser Brauch auf ältere Zeit zurückgeht, ist wegen der unklaren Bedeutung des Wortes ' $m$ ' $(t)$ nicht zu klären. Die Ägypter, sowohl Kopten wie Muslime, haben diesen heidnischen Brauch beibehalten. In Arabien ist er in heidnischer Zeit bekannt, wurde aber von Mohamed weder empfohlen noch verboten. Ob er sich von Ägypten in die Nachbarländer ausgebreitet hat, ist nicht mit Sicherheit festzustellen. Sowohl die Christen wie die Muslime haben ihn als Übernahme aus dem Judentum zu erklären versucht. Allerdings ist auch dies nicht sicher.

Ich fürchte, mit diesem Beitrag mehr Fragen aufgeworfen als beantwortet zu haben. Doch zeigt dies wieder einmal, wie vorsichtig wir bei der Interpretation von Quellen vorgehen müssen. Dem Jubilar wünsche ich noch viele fruchtbare Jahre, die unserer Wissenschaft von Nutzen sein mögen. 